\title{
Asymptotic and Numerical Approximation of a Nonlinear Singular Boundary Value Problem
}

N.B. KONYUKHOVA ${ }^{1}$, Computing Center of RAS, Vavilov Str. 40, 119991 Moscow, Russia

P.M. LIMA2, M.P. CARPENTIER, Centro de Matemática Aplicada, Instituto Superior Técnico, Av. Rovisco Pais 1, 1049-001 Lisboa, Portugal.

Abstract. In this work, we consider a singular boundary value problem for a nonlinear second-order differential equation of the form

$$
g^{\prime \prime}(u)=u g(u)^{q} / q
$$

where $0<u<1$ and $q$ is a known parameter, $q<0$. We search for a positive solution of $(0.1)$ which satisfies the boundary conditions

$$
\begin{gathered}
g^{\prime}(0)=0, \\
\lim _{u \rightarrow 1^{-}} g(u)=\lim _{u \rightarrow 1^{-}}(1-u) g^{\prime}(u)=0 .
\end{gathered}
$$

We analyse the asymptotic properties of the solution of (0.1)-(0.3) near the singularity, depending on the value of $q$. We show the existence of a one-parameter family of solutions of equation (0.1) which satisfy the boundary condition (0.3) and obtain convergent or asymptotic expansions of these solutions.

\section{Introduction}

Let us consider the following nonlinear second-order ordinary differential equation:

$$
g^{\prime \prime}(u)=u g(u)^{q} / q,
$$

where $q<0$ is a known parameter. We shall search for a positive solution of (1.1) which satisfies the boundary conditions:

$$
\begin{gathered}
g^{\prime}(0)=0, \\
\lim _{u \rightarrow 1^{-}} g(u)=\lim _{u \rightarrow 1^{-}}(1-u) g^{\prime}(u)=0 .
\end{gathered}
$$

\footnotetext{
${ }^{1}$ nadja@ccas.ru

${ }^{2}$ plima@math.ist.utl.pt

${ }^{3}$ mcarp@math.ist.utl.pt
} 
This problem arises in the study of boundary layer equations for the stationary incompressible flow of a fluid over a semi-infinite flat plane. We assume that the fluid satisfies a power law, that is a relation of the type:

$$
\tau_{x y}=k\left(\frac{\partial u}{\partial y}\right)^{n}
$$

where $\tau_{x y}$ is the shear stress, $u$ is the velocity, $x, y$ are the coordinates on the plane. Particular cases of such fluids are the newtonian fluids $(n=1)$, the pseudoplastic fluids $(n<1)$ and dilatant fluids $(n>1)$. Under certain conditions, the equation of one of the components of the shear stress may be reduced to the form (1.1), with $q=-1 / n$ (see [11] and [13] ).

The existence and uniqueness of solution of the problem (1.1)-(1.2)-(1.3) were proved in [13]. Numerical methods for the approximation of the solution were proposed in [11], [9] and [10]. However, a detailed analysis of the behavior of the solutions near the singularity at $u=1$ was not provided in those works. In [4] this and other singular boundary value problems for Emden-Fowler type equations were analysed. The main purpose of the present paper is to study the degeneracy of the equation (1.1) when $u \rightarrow 1^{-}$as the solution satisfies the boundary condition (1.3), for different values of $q<0$.

By expanding the one-parametric set of solutions of the singular Cauchy problem (1.1),(1.3) in convergent or asymptotic series we have been able to solve the boundary value problem (1.1)-(1.2) -(1.3) by the shooting method, that is, the parameter of the set of solutions was chosen from the condition that the solution must satisfy (1.2) at $u=0$.

In the second section we shall derive the asymptotic and convergent expansions of the solutions near the singularity, using known results about the singular Cauchy problems (see [5]), smooth manifolds and Lyapunov series (see [6]-[8],[12] and references there).

In the third section we present some numerical results and discuss the properties of the solutions. Finally, in section 4 we present the main conclusions of this work.

\section{Convergent and Asymptotic Expansions}

First of all, let us note the following remarkable fact (see [9] and [10]): when $q=-5$, problem (1.1)-(1.2)-(1.3) has the exact solution

$$
g(u)=10^{-1 / 6}\left(1-u^{3}\right)^{1 / 3}
$$

As we shall see, the asymptotic behavior of the solutions of (1.1)-(1.2) -(1.3) near the singularity depends on the value of $q$. Therefore, we shall consider two cases: $q<-1, q \neq-3$ and $-1<q<0$. Moreover, we shall consider separately the special cases $q=-3$ and $q=-1$. 


\subsection{The Case $q<-1$}

We shall look for a solution of the singular Cauchy problem (1.1),(1.3) in the neighborhood of $u=1$ in the form

$$
\begin{gathered}
g(u)=C(1-u)^{k}[1+o(1)] ; g^{\prime}(u)=-k C(1-u)^{k-1}[1+o(1)] ; \\
g^{\prime \prime}(u)=k(k-1) C(1-u)^{k-2}[1+o(1)], u \rightarrow 1^{-} .
\end{gathered}
$$

From (1.1) and (1.3) it follows that

$$
\lim _{u \rightarrow 1^{-}}\left[g^{\prime \prime}(u) g^{-q}(u)\right]=1 / q<0 .
$$

By substituting (2.2) into (2.3) we obtain

$$
\lim _{u \rightarrow 1^{-}} k(k-1) C^{1-q}(1-u)^{k-2-k q}=1 / q<0,
$$

and therefore

$$
\begin{gathered}
k=\frac{2}{1-q}>0, k-1=\frac{1+q}{1-q}<0, k(k-1)=\frac{2(1+q)}{(1-q)^{2}}<0, \\
C=\left[\frac{(1-q)^{2}}{2 q(1+q)}\right]^{1 /(1-q)}>0 .
\end{gathered}
$$

Let us perform in (1.1) the following change of variable:

$$
g(u)=C(1-u)^{k}[1+y(u)]
$$

where $k$ and $C$ are defined in (2.5). In the new variable, the singular Cauchy problem (1.1),(1.3) may be written as

$$
\begin{gathered}
(1-u)^{2} y^{\prime \prime}-4 \frac{1-u}{1-q} y^{\prime}-\frac{2(1+q)}{(1-q)^{2}}\left((1+y)^{q}-1-y-(1-u)(1+y)^{q}\right)=0,0<u<1, \\
\lim _{u \rightarrow 1^{-}} y(u)=\lim _{u \rightarrow 1^{-}}(1-u) y^{\prime}(u)=0 .
\end{gathered}
$$

For (2.7), $u=1$ is a regular singular point. For solutions that satisfy the conditions (2.8), the leading linear homogeneous terms in (2.7) are

$$
(1-u)^{2} y^{\prime \prime}-4(1-u) y^{\prime} /(1-q)+\frac{2(1+q)}{1-q} y=0, \quad u \approx 1^{-} .
$$

The characteristic exponents of this equation at $u=1$ have opposite signs:

$$
\lambda_{1}=-1, \quad \lambda_{2}=-2 \frac{1+q}{1-q}>0 .
$$

Note that $0<\lambda_{2}<1$ when $-1>q>-3$ and $1<\lambda_{2}<2$ when $q<-3$. Note also that in the special case $q=-3$, we have $\lambda_{2}=1$ and $\lambda_{2}-\lambda_{1}=2$ is an integer. In each of these cases, problem (2.7)-(2.8) has a one-parameter family of solutions. Let us find these families. 
Let us first consider the case $q \neq-3$. First, let us note that problem (2.7)-(2.8) has a particular solution $y_{\text {par }}(u)$ which is holomorphic at $u=1$ :

$$
y_{p a r}(u)=\sum_{k=1}^{\infty} y_{k}(1-u)^{k},|1-u| \leq \delta, \delta>0,
$$

where the coefficients $y_{k}$ can be determined by formal substitution of (2.11) into (2.7); in particular

$$
y_{1}=-\frac{1+q}{(1-q)(3+q)} .
$$

The one-parameter family of solutions to (2.7) that yields $y_{p a r}(u)$ when $a=0$ may be represented in the form

$$
y(u, a)=y_{1}(1-u)+O\left((1-u)^{2}\right)+a(1-u)^{\lambda_{2}}[1+o(1)], u \rightarrow 1^{-},
$$

where $a$ is a parameter, $\lambda_{2}$ and $y_{1}$ are defined by (2.10) and (2.12),respectively. As a result, we have a convergent series written in its general form as

$$
\begin{gathered}
y(u, a)=y_{p a r}(u)+a(1-u)^{\lambda_{2}}\left[1+a_{1}(1-u)+a_{2}(1-u)^{2}+\ldots\right]+ \\
a^{2}(1-u)^{2 \lambda_{2}}\left[b_{0}+b_{1}(1-u)+b_{2}(1-u)^{2}+\ldots\right]+\ldots,|1-u| \leq \Delta(a) .
\end{gathered}
$$

Here $\Delta(a)$ is a certain positive number. In summary, we have the following proposition.

Proposition 1. For any fixed $q$, such that $q<-1$ and $q \neq-3$, the singular Cauchy problem $(1.1),(1.3)$ has a one-parameter family of solutions $g(u, a)$ that can be represented as

$$
\begin{gathered}
g(u, a)=\left[\frac{(1-q)^{2}}{2 q(1+q)}\right]^{\frac{1}{(1-q)}}(1-u)^{\frac{2}{(1-q)}} \\
\times\left\{1-\frac{(1+q)}{(1-q)(3+q)}(1-u)+a(1-u)^{\frac{-2(1+q)}{(1-q)}}+O\left((1-u)^{1+\mu}\right)\right\}, u \rightarrow 1^{-},
\end{gathered}
$$

where $a$ is a parameter and $\mu=\min \left(1, \frac{-2(1+q)}{(1-q)}\right)$. For solutions of this family, $g^{\prime}(u, a)$ and $g^{\prime \prime}(u, a)$ are not bounded as $u \rightarrow 1^{-}$. The general form of the expansion (whose convergence radius is $\Delta(a)>0)$, is given by $(2.14),(2.6)$ and (2.5).

To solve boundary value problem (1.1)-(1.2)-(1.3), one must adjust the parameter $a$ in (2.15), so as to satisfy condition (1.3) at the left endpoint. It is technically more convenient to solve problem (2.7)- (2.8) by adjusting $a$ as imposed by the condition

$$
y^{\prime}(0)-\frac{2}{1-q}(1+y(0))=0,
$$

which is obtained by substituting (2.6) into (1.2). Then we use (2.6) to compute $g(u)$, with $C$ and $k$ defined by (2.5). One should change to the independent variable $v=(1-u)^{1 /(1-q)}$ to transform (2.7)-(2.8) into the problem

$$
v^{2} \ddot{y}+(4+q) v \dot{y}-2(1+q)\left[(1+y)^{2}-1-y-v^{1-q}(1+y)^{q}\right]=0,0<v<1,
$$




$$
\begin{gathered}
\lim _{v \rightarrow 0^{+}} y(v)=\lim _{v \rightarrow 0^{+}} v \dot{y}(v)=0, \\
\dot{y}(1)+2(1+y(1))=0,
\end{gathered}
$$

where $\dot{y}=\frac{d y}{d v}$. Then, to obtain a system of two first-order ODEs, one should introduce $z_{1}=y(v)$ and $z_{2}=v y(v)$.

It is easy to verify that the exact solution to $(1.1)$ given by $(2.1)$ for $q=$ -5 is identical to $g_{\text {par }}(u)=(9 / 10)^{1 / 6}(1-u)^{1 / 3}\left(1+y_{\text {par }}(u)\right)$, where $y_{\text {par }}(u)=$ $\left(\frac{u^{2}+u+1}{3}\right)^{1 / 3}-1$. The series expansion of this function is given by $(2.15)$ with $a=0$.

\subsection{The Case $q=-3$}

Consider the case $q=-3$. Here, $k=1 / 2$ and problem (2.7)-(2.8) is written as

$$
\begin{gathered}
(1-u)^{2} y^{\prime \prime}-(1-u) y^{\prime}+\left((1+y)^{-3}-1-y-(1-u)(1+y)^{-3}\right) / 4=0,0<u<1 \\
\lim _{u \rightarrow 1^{-}} y(u)=\lim _{u \rightarrow 1^{-}}(1-u) y^{\prime}(u)=0
\end{gathered}
$$

Since, in this case, we have $\lambda_{1}=-1, \lambda_{2}=1$ (see (2.10)), we search for a oneparameter family of solutions to this problem in the form

$$
\begin{gathered}
y(u, a)=a(1-u)\left(1+b_{1}(1-u)+\ldots\right)+(1-u) \ln (1-u)\left(c_{0}+c_{1}(1-u)+\ldots\right) \\
+(1-u)^{2} \ln ^{2}(1-u)\left(d_{0}+d_{1}(1-u)+\ldots\right)+\ldots
\end{gathered}
$$

where $a$ is a parameter and the coefficients $b_{j}, c_{j}, d_{j}, \ldots$ generally depend on $a$. These coefficients may be computed by formally substituting (2.22) into (2.20). From (2.6) and (2.22) we then obtain the following result (see [12], [4]-[8] and references there).

Proposition 2. When $q=-3$, the singular Cauchy problem (1.1),(1.3) has a one-parameter family of solutions $g(u, a)$ and the following asymptotic expansion is valid

$$
\begin{gathered}
g(u, a)=\left(\frac{4}{3}\right)^{\frac{1}{4}} \sqrt{1-u}\left\{1-\frac{1}{8}(1-u) \ln (1-u)+a(1-u)-\frac{(1-u)^{2} \ln ^{2}(1-u)}{128}\right. \\
\left.-(1-u)^{2} \ln (1-u)\left(\frac{a}{8}+\frac{1}{96}\right)+(1-u)^{2}\left(\frac{11}{576}-\frac{a}{12}-\frac{a^{2}}{2}\right)+o\left((1-u)^{2}\right)\right\}, u \rightarrow 1^{-},
\end{gathered}
$$

where $a$ is a parameter. The general form of the expansion is given by $(2.22),(2.6)$ and (2.5).

When shooting in the parameter $a$, one should again solve problem (2.17)-(2.18)(2.19), with $q=-3$.

\subsection{The Case $-1<q<0$}

In the neighborhood of $u=1$, the solution to problem (1.1)-(1.2)-(1.3) is sought in the form

$$
\begin{gathered}
g(u)=C_{1}(1-u)+C_{2}(1-u)^{k}(1+o(1)), \\
g^{\prime}(u)=-C_{1}-k C_{2}(1-u)^{k-1}(1+o(1)), \\
g^{\prime \prime}(u)=k(k-1) C_{2}(1-u)^{k-2}(1+o(1)),
\end{gathered}
$$


where $C_{1}>0$ and $k>1$. Combining (2.24) with (2.3), we obtain

$$
\lim _{u \rightarrow 1^{-}} k(k-1) C_{2} C_{1}^{-q}(1-u)^{k-2-q}\left(1+\frac{C_{2}}{C_{1}}(1-u)^{k-2}\right)^{-q}=q^{-1}<0,
$$

which implies

$$
k=2+q>1, C_{2} C_{1}^{-q}=(q(1+q)(2+q))^{-1}<0 .
$$

With $C_{2}=a<0$, the last inequality yields

$$
C_{1}=(a q(1+q)(2+q))^{1 / q}>0 .
$$

Next, we define a function $y(u)$ by

$$
g(u)=C_{1}(1-u)+a(1-u)^{k}(1+y(u)),
$$

where $a<0$ is a parameter, $k$ and $C_{1}$ are defined in (2.26) and (2.27). Then $y(u)$ satisfies the following singular Cauchy problem

$$
\begin{gathered}
(1-u)^{2} y^{\prime \prime}-2(2+q)(1-u) y^{\prime}+(2+q)(1+q) y \\
=(2+q)(1+q)\left[\left(1+a[a q(2+q)(1+q)]^{-1 / q}(1-u)^{1+q}(1+y)\right)^{q}-1\right. \\
\left.-(1-u)\left(1+a[a q(2+q)(1+q)]^{-1 / q}(1-u)^{1+q}(1+y)\right)^{q}\right], 0<u<1, \\
\lim _{u \rightarrow 1-0} y(u)=\lim _{u \rightarrow 1-0}(1-u) y^{\prime}(u)=0 .
\end{gathered}
$$

For each constant $a<0$, this problem has a particular solution $y_{\text {par }}(u, a)$ that can be represented as a convergent series

$$
\begin{aligned}
& \qquad y_{\text {par }}(u, a)=(1-u)^{1+q} \sum_{l=0}^{\infty} y_{l}(1-u)^{l}, 0 \leq 1-u \leq \delta(a), \quad \delta(a)>0, \\
& \text { where } \quad y_{0}(a)=\frac{a(2+q)(a q(2+q)(1+q))^{-1 / q}}{2(3+2 q)} .
\end{aligned}
$$

Let us write the leading linear homogeneous terms in (2.29) for solutions that satisfy (2.30):

$$
(1-u)^{2} y^{\prime \prime}-2(2+q)(1-u) y^{\prime}+(2+q)(1+q) y=0, u \approx 1^{-} .
$$

The characteristic exponents of this equation at $u=1$ are $\lambda_{1}=1-k=-1-q<$ $0, \lambda_{2}=-k=-2-q<0$. Then we conclude (e.g. following [5] ) that for each fixed $a<0$ the singular Cauchy problem (2.29)-(2.30) does not have any solution other than $y_{\text {par }}(u, a)$. In summary, we have the following proposition.

Proposition 3. For any constant $q$ such that $-1<q<0$, the singular Cauchy problem $(1.1),(1.3)$ has a one-parameter family of solutions that can be represented as

$$
\begin{aligned}
& g(u, a)=(a q(2+q)(1+q))^{1 / q}(1-u) \\
& +a(1-u)^{2+q}\left(1+y_{0}(a)(1-u)^{1+q}+O\left((1-u)^{2+q}\right)\right), u \rightarrow 1^{-},
\end{aligned}
$$

where $a<0$ is a parameter and $y_{0}(a)$ is defined by (2.32). The general form of the convergent for $0 \leq 1-u \leq \delta(a)$ expansion is defined by (2.31)-(2.32), (2.28) and (2.26)-(2.27). 


\subsection{The Case $q=-1$}

We seek a solution to problem (1.1)-(1.2)-(1.3) in the form

$$
g(u)=C(1-u) \sqrt{-\ln (1-u)}(1+o(1)), u \rightarrow 1^{-} .
$$

Combining (2.35) with (2.3), we obtain $C=\sqrt{2}$. (The fact that the solution to problem (1.1),(1.3) with $q=-1$ at $u \rightarrow 1^{-}$behaves as $g(u)=\sqrt{2}(1-u) \sqrt{-\ln (1-u)}$ was stated, for example, in [1],p.171).

Next, we define a new unknown function $y(u)$ by the formula

$$
g(u)=\sqrt{2}(1-u) \sqrt{-\ln (1-u)}(1+y(u)), \quad u \rightarrow 1^{-} .
$$

From (1.1) and (1.3), we derive the singular Cauchy problem for $y(u)$

$$
\begin{gathered}
(1-u)^{2} y^{\prime \prime}-2(1-u)[1+1 /(2 \ln (1-u))] y^{\prime}+1 /(2 \ln (1-u))[1-1 /(2 \ln (1-u))] y \\
=1 /\left(4 \ln ^{2}(1-u)\right)+[1 /(2 \ln (1-u))]\left[(1+y)^{-1}-1-(1-u)(1+y)^{-1}\right],(2.37) \\
\lim _{u \rightarrow 1-0} y(u)=\lim _{u \rightarrow 1-0}(1-u) y^{\prime}(u)=0 .
\end{gathered}
$$

The difficulty of this problem lies on the fact that the singular point $u=1$ of $(2.37),(2.38)$ is not regular and it is difficult to determine a linear ODE that governs the essential behavior of solutions to problem (2.37)-(2.38). To deal with this situation, we define a new independent variable

$$
\tau=\sqrt{-\ln (1-u)} .
$$

Denoting $y(u(\tau))$ again by $y(\tau)$, for $0<\tau<\infty$, we can rewrite $(2.37)$ as

$$
\begin{gathered}
\tau^{2} \ddot{y}+\tau \dot{y}\left(1-2 \tau^{2}\right)-\left(1+4 \tau^{2}\right) y=1-2 \tau^{2}\left[(1+y)^{-1}-1+y-\exp \left(-\tau^{2}\right)(1+y)^{-1}\right], \\
\lim _{\tau \rightarrow \infty} y(\tau)=\lim _{\tau \rightarrow \infty} \dot{y}(\tau) / \tau=0 .
\end{gathered}
$$

A particular solution $y_{\text {par }}(\tau)$ to problem $(2.40),(2.41)$ is sought ( by neglecting the term with $\left.\exp \left(-\tau^{2}\right)\right)$ in the form

$$
y_{p a r}(\tau)=\frac{b_{1}}{\tau^{4}}+\frac{b_{2}}{\tau^{6}}+\ldots+\ln \tau\left(\frac{c_{1}}{\tau^{2}}+\frac{c_{2}}{\tau^{4}}+\ldots\right)+\ln ^{2} \tau\left(\frac{d_{1}}{\tau^{4}}+\frac{d_{2}}{\tau^{6}}+\ldots\right)+\ldots
$$

Problem (2.40),(2.41) may be analysed in detail using techniques described in [2], [3] and [14] . As a result, we find that problem (2.40)-(2.41) has a one-parameter family of solutions that can be represented as

$$
y(\tau, a)=y_{p a r}(\tau)+a / \tau^{2}+o\left(1 / \tau^{2}\right), \tau \rightarrow \infty,
$$

where $a$ is a parameter and $y_{\text {par }}(\tau)$ has the form (2.42). Seeking an expansion of this family similar to (2.42) and changing back to the original variable $u$, we finally have the following proposition. 
Proposition 4. When $q=-1$, the singular Cauchy problem (1.1), (1.3) has a one-parameter family of solutions and the following asymptotic representation is valid:

$$
\begin{aligned}
g(u, a)= & \sqrt{2}(1-u) \sqrt{-\ln (1-u)}\left\{1+\frac{\ln (-\ln (1-u)) / 4-a}{\ln (1-u)}\right. \\
& \left.-\frac{\ln ^{2}(-\ln (1-u)) / 32+(1 / 8+a / 4) \ln (-\ln (1-u))-\left(3 / 8+a / 2+a^{2} / 2\right)}{\ln ^{2}(1-u)}+\ldots\right\},
\end{aligned}
$$

where $a$ is a parameter.

It should be noted that the derivation of the representation (2.44) required the manipulation of very long and complicated algebraic expressions, which was possible thanks to the help of a program in the well-known language Mathematica [15].

\section{Numerical Results}

In problem(1.1)-(1.2)-(1.3) the quantity $g(0)$ is an important physical characteristic, proportional to the drag induced by the plate edge. Table 1 shows the values of $a$ and $g(0)$ in problem (1.1)- (1.2)-(1.3) with $-1 \leq q<0$, where $a$ is the parameter of expansion (2.34), when $-1<q<0$, and (2.44), if $q=-1$. These expansions were computed at $u=1-\delta$ where $\delta=0.001$. In Figure 1, the graphics of $g(0)$ and $a$ are displayed, as functions of $-q$.

Note that since expansions (2.15),(2.34) and (2.44) are complicated functions of the parameter $a$, a reasonable value of $\delta$ should be chosen in such a way that a change in $a$ will affect $g(0)$. The values displayed in the tables were obtained with $\delta=0.001$. Analysing the numerical results obtained with different $\delta$, the optimal $\delta$ for most of the considered values of $q$ seems to be in the range $[0.001,0.01]$. If $\delta$ is too small, then the calculation of $a$ may be unstable. This is illustrated by the following example. For $q=-5$, when the exact solution is known and corresponds to $a=0$, the following results were obtained:

\begin{tabular}{|c|c|c|c|c|}
\hline$\delta$ & 0.1 & 0.01 & 0.001 & 0.0001 \\
\hline$a$ & 0.00048 & -0.00012 & -0.0026 & -0.0536 \\
\hline
\end{tabular}

However, our calculations have shown that this unstability does not affect the numerical approximation of the solution (small errors arising in the expansions at $u=1-\delta$ do not grow any further as the Cauchy problem is solved leftwards).

In all the cases where the values of $q$ considered in this study were also considered in [9], the corresponding values of $g(0)$ are also consistent within four or five digits, even though the present computational method is simpler as compared with the previously known ones (see comparisons of different methods in [9],[10]). However, this is achieved by deriving expansions $(2.15),(2.34)$ and $(2.44)$, which are not so simple.

All the results presented in this section were obtained in a PC with a Pentium processor, with the help of programs in Mathematica. These programs used some standard functions of this language, such as NDSolve, which solves numerically a given non-singular Cauchy problem. 


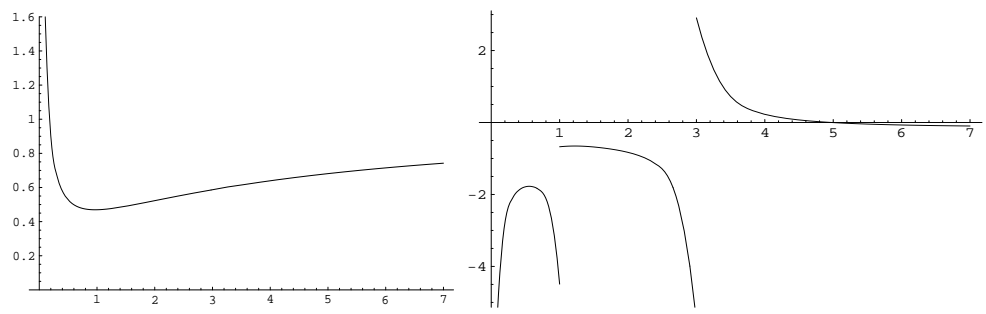

Figure 1: The value of $g(0)$ (on the left) and $a$ (on the right) as functions of $-q$.

\begin{tabular}{|c|c|c|}
\hline$-q$ & $a$ & $g(0)$ \\
\hline 0.1 & -4.96377 & 1.63171 \\
0.2 & -2.78562 & 0.899837 \\
0.3 & -2.14788 & 0.67857 \\
0.4 & -1.88818 & 0.579558 \\
0.5 & -1.78695 & 0.527754 \\
0.6 & -1.78245 & 0.498813 \\
0.7 & -1.87045 & 0.482512 \\
0.8 & -2.1142 & 0.473849 \\
0.9 & -2.86952 & 0.470073 \\
1.0 & -0.267 & 0.46960 \\
\hline
\end{tabular}

Table 1. Numerical results in the case $-1 \leq q<0$.

\section{Concluding Remarks}

The purpose of this paper was to obtain additional analytical results for a wellknown problem in fluid mechanics. This problem illustrates the diversity of situations and complexity of solution behavior characteristic of singular problems for second-order nonlinear ODEs, like (1.1). These results were also used in the numerical solution of the problem and in graphic illustrations of the propositions stated here. In the present study we did not intend to optimize the algorithms for the calculation of the solution to problem. Actually, we have discussed this problem as an example of the correct formulation of singular problems for ODEs and the construction of analytical-numerical methods for their investigation.

\section{Acknowledgements}

The authors acknowledge the support of the NATO project PST/CLG.976878. The first author was also supported by the RFBR projects 99-01-00331 and 02-01-0005.

Resumo. Neste trabalho, consideramos um problema de valores de fronteira singular para uma equação diferencial não-linear de segunda ordem da forma (1.1), onde $0<u<1$ e $q$ é um parâmetro conhecido, $q<0$. Procuramos uma solução positiva 
de (1.1) que satisfaça as condições de fronteira (1.2) e (1.3). Analisamos as propriedades assintóticas da solução de (1.1)-(1.3), consoante o valor de $q$. Provamos a existência de uma família uniparamétrica de soluções da equação (1.1) que satisfazem a condição (1.2) e obtemos desenvolvimentos convergentes ou assintóticos dessas soluções.

\section{References}

[1] C.M. Bender and S. Orszag, "Advanced Methods for Scientists and Engineers", New York, Mc Graw-Hill, 1978.

[2] E.S. Birger and N.B. Lyalikova (Konyukhova), On finding the solutions for a given condition at infinity of certain systems of ordinary differential equations,II, U.S.S.R. Comput. Maths. Math. Phys., 6 (1966), N.3, 47-57.

[3] E.A. Coddington and N.Levinson, "Theory of Ordinary Differential Equations", Mc Graw-Hill, New York, 1955.

[4] A.L. Dyshko, M.P. Carpentier, N.B. Konyukhova and P.M. Lima, Singular problems for Emden-Fowler-type second-order nonlinear ordinary differential equations, Comp. Maths. Math. Phys., 41 (2001), N.4 ,557-580.

[5] N.B.Konyukhova, Singular Cauchy problems for systems of ordinary differential equations, U.S.S.R. Comput. Maths. Math. Phys., 23 (1983), N.3, 72-82.

[6] N.B. Konyukhova, On numerical isolation of the solutions tending do zero at infinity of certain two-dimensional non-linear sets of ordinary differential equations, U.S.S.R. Comput. Maths. Math. Phys., 10 (1970), N.1, 95-111.

[7] N.B. Konyukhova, Stationary Lyapunov problem for a system of first-order quasilinear partial differential equations, Diff. Eq., 30 (1994), N.8, 1284-1294.

[8] N.B. Konyukhova, Stable Lyapunov manifolds for autonomous systems of nonlinear ordinary differential equations, Comput. Maths Math. Phys., 34 (1994), N.10, 1179-1195.

[9] P.M. Lima and M.P. Carpentier, Iterative methods for a singular boundaryvalue problem, J. Comp. Appl. Math.,111 (1999) 173-186.

[10] P.M. Lima and M.P. Carpentier, Numerical solution of a singular boundaryvalue problem in non-Newtonian fluid mechanics, Comp. Phys. Commun., 126 (2000), N.1/2, 114-120.

[11] C.D. Luning and W.L. Perry, An iterative method for solution of a boundary value problem in non-newtonian fluid flow J. Non-Newtonian Fluid Mechanics, 15 (1984), 145-154.

[12] A.M. Lyapunov, "Problème Général de la Stabilité du Mouvement", Kharkov, 1892; reprint, GITTL, Moscow, 1950; French. transl. Ann.Fac.Sci.Univ.Toulouse (2) 9 (1907), 203-474; reprint Ann.of Math. Studies, vol.17, Princeton Univ.Press, Princeton, N.J.,1947.

[13] A. Nachman and A. Callegari, A nonlinear singular boundary value problem in the theory of pseudoplastic fluids, SIAM J. Appl. Math., 38 (1980), 275-281.

[14] W. Wasov, "Asymptotic Expansions for Ordinary Differential Equations", New York, Wiley, 1965.

[15] S. Wolfram, "The Mathematica Book", Cambridge Univ. Press, 1996. 